\title{
Could peptide-decorated nanoparticles provide an improved approach for treating pregnancy complications?
}

\author{
"While the global drug market continues to fail pregnant \\ women, significant steps are now being taken to address \\ the problem and offer creative new solutions."
}

First draft submitted: 10 June 2016; Accepted for publication: 21 June 2016; Published online: 4 August 2016

Keywords: drug delivery $\bullet$ homing peptides $\bullet$ liposomes $\bullet$ nanoparticles $\bullet$ obstetrics - placenta $\bullet$ pregnancy $\bullet$ targeting

\section{Treatment of pregnancy complications: challenges}

Serious pregnancy complications such as preeclampsia, fetal growth restriction (FGR) and preterm labor remain a problem in the western world, despite adequate nutrition and advances in antenatal care. More than $10 \%$ of pregnant women are affected by these conditions, which can cause maternal deaths, stillbirth and neurodevelopmental disorders in surviving infants. Suboptimal development in utero also impacts heavily on health in later life: affected offspring are at increased risk of cardiovascular disease, diabetes and other chronic diseases, both in childhood and adulthood [1,2]. Despite the prevalence and severity of these conditions there are no drug treatments available, leaving clinicians reliant on early delivery, expensive neonatal intensive care and causing further adverse health outcomes in affected children.

The current drug drought in obstetric therapeutics is largely a result of the thalidomide and diethylstilboestrol scandals of the 1950s and 1960s, when off label usage resulted in teratogenic effects. Thalidomide, prescribed to prevent morning sickness, caused severe limb deformities and other developmental abnormalities; diethylstilboestrol, a supposed antimiscarriage drug, induced uterine malformations, infertility and vaginal adenocarcinoma in female babies. The costly lawsuits that ensued led the pharmaceutical industry to consider pregnant women as a high risk, low return cohort, not worthy of further investment. Moreover, the high cost of reproductive toxicology studies, the perceived small market size and endemic off-label prescribing of drugs during pregnancy has discouraged further investment in this area. As a consequence, development of new drugs for use in pregnancy is severely lacking. A 2008 review highlighted that only three drugs had been licensed for use in the last 20 years, two of which are administered after delivery [3]. Only 17 drugs were under active development for maternal health indications, representing less than $3 \%$ of the drug pipeline for cardiovascular disease and fewer compounds than for amyotrophic lateral sclerosis, which only affects $0.004 \%$ of individuals. A more recent analysis showed that only $1.5 \%$ of interventional drug trials were pregnancyrelated, the majority of which are funded by small companies and university-affiliated academics with limited resources [4].

While the global drug market continues to fail pregnant women, significant steps are now being taken to address the problem and offer creative new solutions. Guided by positive results from animal studies, a successful approach has been to repurpose drugs licensed for other indications, using them to increase uteroplacental blood flow and/or treat the placental dysfunction that is characteristic of pre-eclampsia and FGR [5]. Pravas-

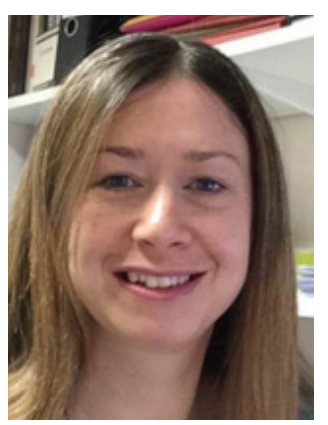

Lynda K Harris

Manchester Pharmacy School, University of Manchester, Stopford Building, Oxford Road, Manchester, M13 9PT, UK and

Maternal \& Fetal Health Research Centre, Institute of Human Development, University of Manchester, Oxford Road, Manchester, M13 9WL, UK and

Academic Health Science Centre, St Mary's Hospital, Oxford Road, Manchester, M13 9WL, UK

lynda.k.harris@manchester.ac.uk 
tatin has been shown to ameliorate preeclampsia and normalize fetal development in pregnant mice $[6,7]$ and the StAmP trial is currently underway to assess its efficacy in humans. Similarly, sildenafil citrate enhanced fetal growth, placental development and umbilical blood flow in pregnant mice and rabbits [8,9], and is now being tested as a treatment for FGR in humans, as part of the STRIDER trial. However, detrimental side effects were reported for both compounds during preclinical testing, including inhibition of placental cell growth [10] and altered fetal vascular function: maternal sildenafil treatment caused cerebral blood overflow in rabbit fetuses [8] and significantly reduced dilatation of mouse fetal aortas in response to vasoactive agonists [11]. Thus, it is imperative to identify and develop therapies that improve maternal and fetal outcomes in the short term, but do not do so at the expense of offspring health in later life.

\section{How could nanomedicines help?}

A major challenge for developing safe medicines for use in pregnancy is to minimize fetal exposure; one way to achieve this would be to selectively deliver the drug to its site of action. Nanoparticle formulations can facilitate this: chemical compounds, biologics and nucleic acid payloads may all be encapsulated or embedded within the core of a nanoparticle, whose properties can then be engineered to target the organ or tissue of interest. This is achieved by manipulation of size and surface charge, incorporation of stealth moieties or by addition of cell-penetrating peptides or targeting elements, such as surface peptides, antibodies or aptamers. As targeted drug delivery has the potential to reduce the risks and side effects associated with systemic drug administration, we hypothesized that targeted nanoparticles could be exploited to treat pregnancy complications.

\section{"As our ultimate aim was to enhance placental growth and optimize pregnancy outcome, we selected liposomes as our nanocarrier of choice for placental targeting."}

The primary cause of pre-eclampsia and FGR is a poorly growing or poorly functioning placenta. Our novel approach to treatment has been to develop placental-specific therapeutics, as there is now a wealth of evidence that enhancement of placental function can improve fetal growth and survival, and alleviate maternal symptoms [5]. By selectively delivering drugs to this temporary organ of pregnancy, we aimed to maximize drug efficacy and minimize off-target side effects in maternal and fetal tissues. As the developing placenta shares many characteristics with solid tumors [12], including the ability to proliferate rapidly, invade the uterus, alter the local vasculature and influence immune cell function, we postulated that previously identified tumor homing peptides would also selectively bind to the placenta. We confirmed this to be the case [13]; following intravenous injection, the tumor homing sequences CGKRK and CRGDKGPDC (iRGD) localized to the placentas and uterine spiral arteries of pregnant mice and multiple injections during pregnancy had no adverse effect on placental function or pregnancy outcome. The peptides also bound to explants of human placental tissue, accumulating in the outermost syncytiotrophoblast cell layer, without adversely affecting placental cell turnover. Having identified suitable targeting moieties, we then sought to create functional nanocarriers.

The majority of targeted nanomedicines have been developed deliver chemotherapeutics to tumors; in this field, biocompatibility of the nanoparticle is of lesser importance when the ultimate aim is to kill the target cell. Until recently, relatively little was known of the fate of nanoparticles in pregnancy models, nor how placental transit is regulated. A number of studies, primarily undertaken in rodents, have now demonstrated that particle size, surface charge and composition all influence rate and extent of placental uptake, fetal transfer and associated fetal toxicity (reviewed in [14]). However, many of the formulations examined (e.g., polystyrene, quantum dots, silica dioxide, carbon nanotubes and silver, gold, titanium oxide- and iron oxide nanoparticles) would not be considered compatible with maintenance of normal placental function. As our ultimate aim was to enhance placental growth and optimize pregnancy outcome, we selected liposomes as our nanocarrier of choice for placental targeting. As observed with free peptide, liposomes decorated with the CGKRK or iRGD sequences bound to, and accumulated within the syncytiotrophoblast layer of human placental explants, and also selectively bound to the placental labyrinth and the uterine spiral arteries when injected intravenously into pregnant mice [13]. Liposomes decorated with control peptides, or bearing no surface peptides accumulated in human and mouse placental tissue at far lower levels. By encapsulating a fluorophore inside the liposomes to represent a drug payload, we showed that targeted liposomes delivered and released their cargo within the mouse placenta, with no transfer to the fetus. We then utilized this liposomal formulation to administer IGF-II, a molecule known to enhance placental growth and function. Targeted placental delivery of IGF-II significantly increased placental weight in healthy pregnant mice, but placental weight remained unchanged in mice treated with free IGF-II, empty liposomes or nontargeted liposomes containing IGF-II. Surprisingly, fetal 
weight was not significantly increased by targeted placental delivery of IGF-II as we had predicted, perhaps because these healthy fetuses were already growing at an optimal rate. We therefore investigated whether targeted delivery of IGF-II could enhance fetal growth in the placental-specific Igf-II knockout mouse, a well characterized model of FGR. Compared with controls, targeted placental delivery of IGF-II increased the weight of the smallest fetuses within each litter, so that none of the offspring fell below the 10th centile in weight. Importantly, this treatment did not increase the weight of the larger pups, suggesting that it acts only on the poorly growing fetuses that are at most need of an intervention, a clinically relevant outcome.

\section{Novel uteroplacental delivery systems}

Several research groups are now beginning to develop similar nanomedicines to treat pregnancy complications: in the first study of its kind, Kaitu'u-Lino et al. created a nanoformulation to treat ectopic pregnancy and placental-derived tumors, using bacterially derived nanocells decorated with antibodies to the EGF receptor, a molecule that is highly expressed on the placental surface [15]. When loaded with doxorubicin, these nanoparticles inhibited the growth of the placental choriocarcinoma cell line JEG-3 in vitro and limited the size of JEG-3 tumor xenografts in vivo, significantly more effectively than nontargeted nanoparticles or free doxorubicin. Polymeric nanocomplexes containing a human IGF-1 plasmid under the control of trophoblastspecific promoters have been exploited to induce IGF-1 transgene expression in a placental cell line, with the aim of enhancing growth and survival [16]. Direct injection of these complexes into mouse placentas resulted in transgene expression and significantly increased fetal weights in a model of FGR. In an approach most similar to our own, liposomes decorated with antibodies to the oxytocin receptor have been created for targeted delivery of tocolytics to the pregnant uterus [17]. Targeted liposomes loaded with nifedipine, salbutamol or rolipram inhibited contraction of pregnant uterine tissue in vitro, whereas drug-loaded, nontargeted liposomes had no effect. When administered to pregnant mice, control liposomes localized exclusively to the liver, whereas targeted liposomes were also observed in uterine and mammary tissue, and did not cross to the fetus [17]. This formulation shows great promise as a potential therapy for preterm labor.

\section{Future perspective}

Now that methods for targeted uteroplacental drug delivery have been established and validated, this technology can be exploited in a number of ways, including more effective delivery of siRNAs, microRNAs
(miRs) and gene therapies. For example, the EVERREST trial is currently testing whether an adenoviral vector encoding VEGF-D can enhance uterine blood flow and improve fetal growth in severe, early-onset FGR, after promising results in sheep [18]. Administration of such a construct using targeted liposomes may further enhance tissue-specific delivery, cellular uptake and transfection efficiency. We have preliminary evidence that placental homing peptides can be exploited for targeted delivery of a miR-145 inhibitor, leading to enhanced trophoblast proliferation in human placental explants and increased placental and fetal weights in pregnant mice [19]. It is also tempting to speculate whether fetal targeting might be possible; if fetal homing peptides were identified, it may permit delivery of therapies to individual fetal organs or targeted administration of fetal-specific signals that promote growth and survival.

\section{“...exploiting peptide-decorated liposomes for uteroplacental-specific targeting has not only opened new avenues for the development of novel and efficacious obstetric therapeutics, but may also provide a platform to test new drugs more safely.'}

However, numerous important questions remain to be answered before obstetric nanomedicines reach the clinic. First, very little is known about how the maternal immune system handles a nanoparticle challenge in pregnancy. Given that pregnancy induces an immunotolerant state [12], women may not mount a significant response. However, as pre-eclampsia is characterized by immune cell activation, a pro-inflammatory milieu and global maternal endothelial dysfunction, administration of nanoformulations may exacerbate maternal symptoms. Second, longitudinal studies are required to determine whether exposure to liposomes in pregnancy has any long-term effects on the offspring. Although our experiments showed that targeted liposomes did not cross the placenta [13], we cannot rule out the possibility that fetuses were exposed to lipid breakdown products, potentially elevating fetal plasma lipid concentrations above normal levels and eliciting developmental programming effects. It is therefore vital to understand what, if any, impact liposomal therapies have on neonatal, childhood and adult health. Finally, studies investigating liposome uptake and transfer across perfused human term placental lobules have been undertaken and showed that size and surface charge are important determinants of transplacental passage [20]. However, the pharmacokinetics of peptide-decorated liposomes may be quite different, and represent a worthy area of future study using this unique experimental model. 
In summary, exploiting peptide-decorated liposomes for uteroplacental-specific targeting has not only opened new avenues for the development of novel and efficacious obstetric therapeutics, but may also provide a platform to test new drugs more safely. In the longer term, our hope is that this technology has the potential to stimulate interest and investment by the pharmaceutical industry, in turn addressing the longstanding drug drought in obstetric care.

\section{References}

1 Gluckman PD, Hanson MA, Cooper C, Thornburg KL. Effect of in utero and early-life conditions on adult health and disease. N. Engl. J. Med. 359(1), 61-73 (2008).

2 Davis EF, Lazdam M, Lewandowski AJ et al. Cardiovascular risk factors in children and young adults born to preeclamptic pregnancies: a systematic review. Pediatrics 129(6), e1552-e1561 (2012).

3 Fisk NM, Atun R. Market failure and the poverty of new drugs in maternal health. PLoS Med. 5(1), e22 (2008).

4 Keelan JA, Leong JW, Ho D, Iyer KS. Therapeutic and safety considerations of nanoparticle-mediated drug delivery in pregnancy. Nanomedicine 10 (14), 2229-2247 (2015).

5 Spencer RN, Carr DJ, David AL. Treatment of poor placentation and the prevention of associated adverse outcomes - what does the future hold? Prenat. Diagnos. 34(7), 677-684 (2014).

6 Kumasawa K, Ikawa M, Kidoya $\mathrm{H}$ et al. Pravastatin induces placental growth factor (PGF) and ameliorates preeclampsia in a mouse model. Proc. Natl Acad. Sci. USA 108(4), 1451-1455 (2011).

7 McDonnold M, Tamayo E, Kechichian T et al. The effect of prenatal pravastatin treatment on altered fetal programming of postnatal growth and metabolic function in a preeclampsia-like murine model. Am. J. Obstet. Gynecol. 210(6), 542.e541-e547 (2014)

8 Lopez-Tello J, Arias-Alvarez M, Jimenez-Martinez MA et al. The effects of sildenafil citrate on feto-placental development and haemodynamics in a rabbit model of intrauterine growth restriction. Reprod. Fertil. Develop. doi:10.1071/RD15330 (2016) (Epub ahead of print).

9 Stanley JL, Andersson IJ, Poudel R et al. Sildenafil citrate rescues fetal growth in the catechol-O-methyl transferase knockout mouse model. Hypertension 59(5), 1021-1028 (2012).

10 Forbes K, Hurst LM, Aplin JD, Westwood M, Gibson JM. Statins are detrimental to human placental development and
Financial \& competing interests disclosure

This work was supported by a BBSRC David Phillips Research Fellowship to LK Harris. The author has no other relevant affiliations or financial involvement with any organization or entity with a financial interest in or financial conflict with the subject matter or materials discussed in the manuscript apart from those disclosed.

No writing assistance was utilized in the production of this manuscript.

function; use of statins during early pregnancy is inadvisable. J. Cell. Mol. Med. 12(6A), 2295-2296 (2008).

11 Renshall LJ, Dilworth MR, Greenwood SL, Sibley CP, Wareing M. In vitro assessment of mouse fetal abdominal aortic vascular function. Am. J. Physiol. 307(6), R746-R754 (2014).

12 Holtan SG, Creedon DJ, Haluska P, Markovic SN. Cancer and pregnancy: parallels in growth, invasion, and immune modulation and implications for cancer therapeutic agents. Mayo Clinic Proc. 84(11), 985-1000 (2009).

13 King A, Ndifon C, Lui $S$ et al. Tumour homing peptides as tools for targeted delivery of payloads to the placenta. Sci. Adv. 2, e1600349 (2016).

14 Muoth C, Aengenheister L, Kucki M, Wick P, BuerkiThurnherr T. Nanoparticle transport across the placental barrier: pushing the field forward! Nanomedicine 11(8), 941-957 (2016).

15 Kaitu'u-Lino TJ, Pattison S, Ye L et al. Targeted nanoparticle delivery of doxorubicin into placental tissues to treat ectopic pregnancies. Endocrinology 154(2), 911-919 (2013).

16 Abd Ellah N, Taylor L, Troja W et al. Development of nonviral, trophoblast-specific gene delivery for placental therapy. PLoS ONE 10(10), e0140879 (2015).

17 Paul J, Hua S, Smith R. A targeted drug delivery system for the uterus. Reprod. Sci. 22 (3, Suppl. 1), 57A (2015).

18 Carr DJ, Wallace JM, Aitken RP et al. Uteroplacental adenovirus vascular endothelial growth factor gene therapy increases fetal growth velocity in growth-restricted sheep pregnancies. Human Gene Ther. 25(4), 375-384 (2014).

19 Beards F, Jones L, Charnock J, Forbes K, Harris LK. Placental homing peptide-microRNA-145 inhibitor conjugates for targeted enhancement of placenta growth. Reprod. Sci. 22(3, Suppl. 1), 77A (2015).

20 Bajoria R, Contractor SF. Effect of surface charge of small unilamellar liposomes on uptake and transfer of carboxyfluorescein across the perfused human term placenta. Pediatr. Res. 42(4), 520-527 (1997). 